\title{
A real-time PCR assay for quantification of parasite burden in murine models of leishmaniasis
}

\author{
Alejandro L Antonia ${ }^{1}$, Liuyang Wang ${ }^{1}$, Dennis C Ko ${ }^{\text {Corresp. }{ }^{1,2}}$ \\ 1 Department of Molecular Genetics and Microbiology, Duke University, Durham, North Carolina, United States \\ 2 Division of Infectious Diseases, Department of Medicine, Duke University, Durham, North Carolina, United States \\ Corresponding Author: Dennis C Ko \\ Email address: dennis.ko@duke.edu
}

Eukaryotic parasites in the genus Leishmania place approximately 350 million people per year at risk of disease. In addition to their global health significance, Leishmania spp. have served as an important model for delineating basic concepts in immunology such as Thelper cell polarization. There have been many qPCR-based assays reported for measuring parasite burden in humans and animals. However, these are largely optimized for use in clinical diagnosis and not specifically for animal models. This has led several of these assays to have suboptimal characteristics for use in animal models. For example, multicopy number genes have been frequently used to increase sensitivity but are subject to greater plasticity within the genome and thus may confound effects of experimental manipulations in animal models. In this study, we developed a sybr-green based quantitative touchdown PCR assay for a highly conserved and single-copy putative RNAbinding protein, DRBD3. With primers that share greater than $90 \%$ sequence identity across all sequenced Leishmania spp., we demonstrate that this assay has a lower limit of detection of $100 \mathrm{fg}$ of parasite DNA for $L$. major, $L$. donovani, $L$. venezuelensis, and $L$. panamensis. Using C57BL6/J mice, we used this assay to monitor parasite burden over 1 month of infection with two strains of $L$. major (Seidman and Friedlin), and $L$. venezeuelensis. These characteristics rival the sensitivity of previously reported qPCR based methods of parasite quantitation while amplifying a stable, single copy gene. Use of this protocol in the future will lead to improved accuracy in animal based models and help to tease apart differences in biology of host-parasite interactions. 
5 A Real-time PCR Assay for Quantification of Parasite Burden in Murine Models of 6 Leishmaniasis

7

8

9

10 Authors:

11 Alejandro L. Antonia ${ }^{1}$, Liuyang Wang ${ }^{1}$, and Dennis C. Ko ${ }^{1,2}$

12 Affiliations:

$13{ }^{1}$ Department of Molecular Genetics and Microbiology, School of Medicine, Duke University, 14 Durham, NC 27710

15 2Division of Infectious Diseases, Department of Medicine, School of Medicine, Duke University,

16 Durham, NC 27710

17 *To whom correspondence should be addressed: dennis.ko@duke.edu.

18

19 


\section{Abstract}

21 Eukaryotic parasites in the genus Leishmania place approximately 350 million people per year at

22 risk of disease. In addition to their global health significance, Leishmania spp. have served as an

23 important model for delineating basic concepts in immunology such as T-helper cell polarization.

24 There have been many qPCR-based assays reported for measuring parasite burden in humans and

25 animals. However, these are largely optimized for use in clinical diagnosis and not specifically for

26 animal models. This has led several of these assays to have suboptimal characteristics for use in

27 animal models. For example, multi-copy number genes have been frequently used to increase

28 sensitivity but are subject to greater plasticity within the genome and thus may confound effects

29 of experimental manipulations in animal models. In this study, we developed a sybr-green based

30 quantitative touchdown PCR assay for a highly conserved and single-copy putative RNA-binding

31 protein, DRBD3. With primers that share greater than $90 \%$ sequence identity across all sequenced

32 Leishmania spp., we demonstrate that this assay has a lower limit of detection of $100 \mathrm{fg}$ of parasite

33 DNA for L. major, L. donovani, L. venezuelensis, and L. panamensis. Using C57BL6/J mice, we

34 used this assay to monitor parasite burden over 1 month of infection with two strains of L. major

35 (Seidman and Friedlin), and L. venezeuelensis. These characteristics rival the sensitivity of

36 previously reported qPCR based methods of parasite quantitation while amplifying a stable, single

37 copy gene. Use of this protocol in the future will lead to improved accuracy in animal based models

38 and help to tease apart differences in biology of host-parasite interactions. 


\section{Introduction}

43

44 45

Parasites in the genus Leishmania cause a spectrum of disease ranging from cutaneous (CL) to visceralizing (VL) disease and are frequently used in many experimental animal models. Up to 1.6 million people each year are infected with one of the forms of leishmaniasis (Alvar, Velez et al. 2012). This significant impact of disease resulted in an estimated 980,000 disability adjusted life years (DALYs) in 2016 (DALYs and Collaborators 2017). Further, these estimates are likely to significantly underestimate the burden caused by social and psychological stigmatization resulting from long term scarring (Bailey, Mondragon-Shem et al. 2017).

The outcome of Leishmania infection has been understood to depend on proper T-helper $\left(T_{h}\right)$ cell polarization since the late 1980's when it was shown that a $T_{h} 1$ response promotes a healing immune response whereas a $\mathrm{T}_{\mathrm{h}} 2$ response leads to progressive, non-healing disease (Scott, Natovitz et al. 1988, Heinzel, Sadick et al. 1989). Since then, animal models of leishmaniasis have continued to be used to characterize and understand many important aspects of the adaptive immune system. Despite these many advances in understanding immunologic concepts broadly, our understanding of the immune response to Leishmania spp. specifically is evolving to reflect greater nuances and complexities (Scott and Novais 2016). Further, current treatment options for leishmaniasis remain prolonged, expensive, have variable efficacy, and significant side effects presenting an urgent need for novel therapeutics (Ponte-Sucre, Gamarro et al. 2017). In order to investigate these effectively, it is paramount to have optimal laboratory techniques for assessing disease progression in animals accurately, reproducibly, and under a variety of experimental conditions.

Methods to monitor disease progression during Leishmania infection in cutaneous animal models have traditionally relied on measuring footpad swelling and using a limiting dilution assay 
65 (LDA) to quantify parasite burden (Sacks and Melby 2001). While LDA remains a reliable and

66 sensitive technique for quantifying parasite burden, these assays are labor intensive, can be

67 technically challenging, and take several weeks to obtain final results. Recent advances employing

68 genetic manipulation of parasites to express live reporter molecules such as mCherry or luciferase

69 allow advanced monitoring of the parasite in real time and over a long-time course; however, they

70 require an additional layer of genetic manipulation on the parasite and often require expensive

71 equipment for visualization (Roy, Dumas et al. 2000, Michel, Ferrua et al. 2011, Calvo-Alvarez,

72 Guerrero et al. 2012). More recently developed protocols utilizing amplification of nucleic acids

73 have been optimized for use in clinical diagnosis. Additionally, novel modifications to PCR

74 based techniques, such as PCR-ELISA, hold significant promise in improving sensitivity and high

75 throughput capacity of parasite detection (Kobets, Badalova et al. 2010). To meet the demands of

76 a clinical diagnostic assay, such as high sensitivity and the ability to discriminate between

77 Leishmania spp., highly variable and multi-copy genes are most commonly used.

78 However, characteristics for clinical assays for Leishmania detection are not necessarily

79 ideal for use in experimental animal models. The ability to differentiate between different strains

80 is not required when the infecting species is carefully controlled and delivered during experimental

81 infection. Additionally, the use of multi-copy genes which are known to be present in regions of

82 relative genomic plasticity, may change during the course of infection. This at best adds

83 unpredictable variation to the assay and at worst confounds the observation by inducing a

84 systematic change across only certain experimental conditions. For instance, a comparative

85 genomic study suggested that the high degree of variability of gene copy number across

86 Leishmania spp. provides a potential mechanism to adapt to environmental change by altering

87 gene expression (Rogers, Hilley et al. 2011). Subsequently experimental studies have confirmed 
88 that Leishmania spp. regulate gene expression by altering gene copy number (Iantorno, Durrant et

89 al. 2017), and that this process can lead to drug resistance (Laffitte, Leprohon et al. 2016). Finally,

90 it is particularly important to select genes without an active role in disease, as any studies to further

91 understand these or related pathways are subject to the risk of mutations or gene copy expansions

92 rendering PCR at these sites unable to accurately compare across experimental conditions.

93 Recently, several efforts have been made to standardize lab protocols for detection and 94 quantification of parasites from clinical isolates (Cruz, Millet et al. 2013, Leon, Munoz et al. 2017).

95 However, similar comparative studies that emphasize assay traits optimized for experimental 96 models of infection are lacking. Here we report a real-time quantitative PCR (qPCR) assay based 97 on amplification of a single-copy, housekeeping Leishmania RNA binding protein (DRBD3) that 98 is optimal for animal model studies. With touchdown cycling parameters, we were able to achieve 99 a sensitivity of $100 \mathrm{fg}$ per reaction which rivals most described PCR protocols for Leishmania 100 quantification. Use of this assay in the future will facilitate studies elucidating mechanisms of 101 immunity to Leishmania spp. and in monitoring efficacy of novel pharmaceutical interventions.

102 Materials and Methods

103 Multisequence alignment and sequence Logo

104 Traits of the DRBD3 gene were analyzed in TriTrypDB using the L. major Fd reference 105 sequence (LmjF.04.1170) (Aslett, Aurrecoechea et al. 2010). The reference sequence used in 106 NCBI Blast tool to identify homologous sequences was based on the L. major SD75.1 sequence in 107 order to correspond with the parasite DNA used to validate the assay in this study. The 11 identified 108 homologous genomic DNA sequences were downloaded and aligned using ClustalOmega 109 (McWilliam, Li et al. 2013). The WebLogo tool was used to generate a sequence logo based on 110 this alignment ( http://weblogo.berkeley.edu/) (Crooks, Hon et al. 2004). 
111

112

113

114

115

116

117

118

119

120

121

122

123

124

125

126

127

128

129

130

131

132

133

Primers were designed using Primer-BLAST software (Ye, Coulouris et al. 2012). The gene for DRBD3 in the Leishmania major (MHOM/SN/74/Seidman) was input. Five pairs of primers with similar melt temperatures were initially tested. After amplification by conventional PCR, 3 of these primer pairs resulted in non-specific amplification as detected by ethidium bromide detection in a 1\% agarose gel. Amplicon size was verified by separating the PCR product in a $1 \%$ agarose gel, visualizing the product by ethidium bromide staining, and comparing to $100 \mathrm{bp}$ DNA staining ladder (New England Bioscience, N3231S). Based on this initial screen, the primers eported in Figure 1B were then used to amplify wildtype L. major (MHOM/SN/74/Seidman) promastigote DNA as described below.

qPCR protocol for parasite quantification

After DNA isolation from each mouse tissue, 100ng of DNA was used per reaction on a calculated using the formula $\left(10^{\left(-\frac{1}{m}\right)}\right)-1$, where $m$ is the slope from a plot of the $C_{t} v s \log (\operatorname{parasite}$ DNA) with serial 10 fold dilutions ranging from $1 \times 10^{7}$ fg to 100 fg total DNA per reaction. 
134 on each 96-well plate. DNA (fg per reaction) was then multiplied by the factor used to dilute each 135 sample to $100 \mathrm{ng}$ per PCR reaction in order to get total parasite DNA (fg) per tissue harvested. 136 Parasites and culture conditions

137 Leishmania major Seidman (Sd.) (MHOM/SN/74/Seidman), Leishmania major Friedlin 138 (Fd.) (MHOM/IL/80/FN), L. donovani (MHOM/6D/62/1S), L. venezuelensis (MHOM/VE/80/H139 16), and L. panamensis (MHOM/PA/94/PSC-1) were obtained from BEI Resources. Parasites 140 were maintained in M199 media supplemented with 10\% heat inactivated FBS and $0.2 \%$ hemin.

141 Cultures were maintained by inoculating a new $10 \mathrm{~mL}$ culture with $200 \mu \mathrm{l}$ of previous culture 142 every 5 days. Infections in mice were performed with parasites that had been passaged less than 3 143 times in vitro.

144 Mouse infections

145 Wildtype C57BL6 mice from the Jackson Laboratory were maintained in the Duke 146 Laboratory Animal Resource (DLAR) Breeding Core. All studies were approved under Duke 147 University IACUC protocol A200-15-07. L. major (MHOM/SN/74/Seidman) parasites were 148 prepared by washing 5 day old culture of promastigotes with Hanks Buffered Salt Solution 149 (HBSS), counting by hemocytometer, and resuspending at $2 \times 10^{6}$ parasties per $50 \mu 1$ of HBSS. A $15027 \mathrm{G} 1 / 2 \mathrm{~mL}$ syringe with permanently attached needle was used to inoculate the left hind footpad 151 with $50 \mu 1$ of promastigote suspension. Mice were monitored at least twice weekly to track lesion 152 development.

153 DNA was obtained using the Qiagen DNeasy Blood and Tissue Kit (Cat \# 69504). In brief, 154 tissue from the infected footpads and draining popliteal lymph node of each mouse was harvested. 155 The contralateral footpad and lymph node was taken from each mouse to monitor for 156 contamination. Tissue was placed into a clean $1.5 \mathrm{~mL}$ microcentrifuge tube. To $180 \mu \mathrm{l}$ of buffer 
157 ATL and $20 \mu \mathrm{l}$ of proteinase $\mathrm{K}$ was added to each tube prior to homogenizing the tissue with a

158 bead beater and incubating samples at $37^{\circ} \mathrm{C}$ overnight before proceeding as indicated by

159 manufacturer instructions. DNA quantity and quality was assessed using the Take3 application on

160 a Synergy H1 BioTek plate reader prior to use in the qPCR assay described. 33 out of 35 uninfected

161 footpads and non-draining lymph nodes included as negative controls, did not produce a

162 leishmania specific product as determined by melt temperature analysis $\left(84.7^{\circ} \mathrm{C}\right)$

163

$164 \underline{\text { Results }}$

$165 q P C R$ assay design for the Leishmania RNA binding protein, DRBD3.

166 To design a qPCR assay with optimal characteristics for quantification of Leishmania

167 parasite burden in experimental models, we first sought to identify a single copy gene essential to

168 the parasite, but less likely to be actively involved in evasion of host defense. For this we chose

169 putative Leishmania RNA binding protein (DRBD3), present in a single-copy on chromosome 4.

170 The gene is classified as the double RNA binding domain 3 protein based on homology with

171 DRBD3 in Trypanosoma brucei. Studies in T. brucei have identified a consensus binding sequence

172 in the 3'UTR of mRNA transcripts and suggest that DRBD3 plays a role in modulating stress

173 response (Fernandez-Moya, Garcia-Perez et al. 2012, Das, Bellofatto et al. 2015); however, there

174 have been no functional studies of this protein in Leishmania spp. to date. The L. major entry for

175 DRBD3 (LmjF.04.1170) on TriTrypDB indicates that the gene is constitutively expressed between

176 parasite life-cycle stages, is not under immune pressure, and has minimal sequence variation

177 between species (Aslett, Aurrecoechea et al. 2010). RNA-seq experiments in both L. major and L.

178 amazonensis have demonstrated little to no change in expression of this gene between the

179 promastigote and amastigote stage consistent with the characteristics of a constitutively expressed 
180 housekeeping gene (Akopyants, Matlib et al. 2004, Leifso, Cohen-Freue et al. 2007, Aoki, Muxel

181 et al. 2017). Additionally, there were no identified epitopes in the Immune Epitope Database

182 (IEDB) corresponding to DRBD3 peptides, suggesting that it is not likely to be influenced by host

183 immune pressure (Vita, Zarebski et al. 2010). To verify the homology of DRBD3 between

184 Leishmania spp. we used the sequence from L. major Sd. as a reference, and used NCBI blast

185 software to identify all related sequences. This blast search yielded 11 hits of protein coding 186 genomic DNA within the genus Leishmania. Using ClustalOmega we then created a multisequence

187 alignment, and in combination with NCBI Primer Blast tool designed primers over a highly188 conserved region (Figure 1A). Both primers have complete conservation at 18 out of 20 189 nucleotides. This design allows using a single set of primers to PCR amplify a stable, housekeeping 190 gene that is likely to be applicable across a broad range of Leishmania spp.

touchdown PCR cycling parameters, one distinct product is visible by ethidium bromide staining,

on a standard curve extending across six orders of magnitude from $1 \times 10^{7} \mathrm{fg}-100 \mathrm{fg}$ of parasite can detect DNA above this, but is no longer within the linear range. Using this same assay, DNA 197 from geographically and phenotypically diverse parasites (L. major Fd., L. venezuelensis, $L$. 198 panamensis, and L. donovani) were then validated and found to have the same limit of 199 quantification (100 fg) and comparable primer efficiency (Figure 1D). Of note, the L. panamensis 200 sequence of DRBD3 has a mismatch at 2 out of 20 base pairs for each primer; however, this did 201 not significantly impair the limit of quantification or primer efficiency. Therefore, we successfully 
202 developed a novel touch-down based PCR assay for a single-copy, housekeeping gene in 203 Leishmania spp. that is able to efficiently detect as low as $100 \mathrm{fg}$ of parasite DNA per reaction.

204

205 Validation of DRBD3 qPCR in vivo

206 We then tested the assay's ability to monitor parasite burden in the murine model of 207 cutaneous leishmaniasis. Wildtype C57BL6 mice were inoculated subcutaneously with $2 \times 10^{6}$ 208 cultured L. major Sd. promastigotes in the left hind footpad. At 1 day and 34 days post infection, 209 DNA was isolated from the infected and uninfected footpads as well as the corresponding popliteal 210 lymph nodes.

The assay was able to accurately monitor parasite burden over this course of infection.

212 Representative amplification and melting temperature plots are shown in Figure $2 \mathrm{~A}$ and B. In 213 uninfected samples, nonspecific products were easily differentiated by a melting temperature 214 outside of the expected $84.7 \pm 0.5^{\circ} \mathrm{C}$. At 1 day post infection, 6 of 7 mice had low, yet detectable 215 parasite burdens in the footpad, but only 2 out of 7 infected mice had detectable parasites in the 216 draining lymph node. This is consistent with an expected delay in time for migration of Leishmania 217 parasites to the draining lymph nodes. In both the draining lymph nodes and infected footpads, a 218 significant increase in parasite burden was observed between 1 day and 34 days post infection, 219 where all samples had leishmania specific amplification (Figure 2C).

220 Having established that the primers work in vivo to monitor L. major Sd. infection, we next 221 validated the assay on the traditional reference strain of $L$. major Fd. and with a strain of $L$. 222 venezuelensis. Measuring footpad thickness with calipers demonstrate that at 36 days post 223 infection there are significantly larger lesions in the L. major Fd. infected mice. However, using 224 the DRBD3 quantitative PCR method to quantify parasite burden revealed that the L. venezuelensis 
225 infected lesions harbored more parasites despite the lower overall degree of inflammation and

226 swelling at the site of the lesion (Figure 2D). The discordance is consistent with previous reports

227 of L. major Fd. successfully clearing parasites, in contrast to infection with new world Leishmania

228 spp. in the leishmania subgenus, such as L. venezuelensis, which results in smaller, chronic and

229 non-healing lesions (Scott and Novais 2016). Therefore, this assay successfully monitors

230 Leishmania spp. parasite burden in mouse models of cutaneous leishmaniasis.

231

232 Comparison of DRBD3 qPCR assay to other targets for quantification of Leishmania spp. infection 233 in animal models

234 We compared the characteristics of the DRBD3 assay to other published qPCR assays. A

235 wide variety of assays for Leishmania detection by PCR with diverse targets and characteristics

236 have been described (Table 1); though, most have been optimized from the perspective of clinical

237 diagnostics.

238

The most commonly used assays target multicopy genes. This produces advantages in

239

regards to lower limits of detection and the potential to discriminate between species but come at

240 the cost of uncertainty in gene stability at these plastic regions of the genome. 18s rRNA is present

241 in up to 166 copies per parasite (Ivens, Peacock et al. 2005, Torres-Machorro, Hernandez et al.

242 2010), the internal transcribed spacer (ITS-1), found in between the small and large rRNA subunits

243 between 40-200 (Cupolillo, Grimaldi Junior et al. 1995, Bensoussan, Nasereddin et al. 2006),

244 minicircle kinetoplast DNA at up to 10,000 copies (Yurchenko, Merzlyak et al. 1999), and HSP70

245 at 5-7 (Folgueira, Canavate et al. 2007). These assays report limits of detection as low as $10 \mathrm{fg}$ of

246 parasite DNA per reaction. However, multicopy regions are often under significant change. For

247 example, kDNA is particularly unstable in terms of copy number with reports of significant 
248 variation between species, strains, lifecycle stages, and clinical isolates (Mary, Faraut et al. 2004,

249 Weirather, Jeronimo et al. 2011, Jara, Adaui et al. 2013).

250 Single copy genes tend to be in more stable regions of the genome, but report higher limits

251 of detection compared to multicopy gene assays and variable efficiencies between studies.

252 Glucosephosphate isomerase (GPI), glucose 6 phosphate dehydrogenase (G6PD), Superoxide

253 Dismutase 1 (SODB1) (Ghotloo, Haji Mollahoseini et al. 2015), Arginine Permease (AAP3)

254 (Tellevik, Muller et al. 2014), and DNA polymerase alpha (Croan, Morrison et al. 1997) are all

255 single copy genes for which Leishmania quantification by PCR has been described. The assay for

256 GPI reports a higher limit of detection of $5.6 \mathrm{pg}$ compared to other assays with limits of detection

257 around 10-100 fg per reaction (Wortmann, Hochberg et al. 2005). Reported PCR efficiencies for

258 the G6PD assays range from 50.4\% to 95.7\%. SODB1 and AAP3 described qPCR assays have

259 competitive limits of detection and efficiencies (Tellevik, Muller et al. 2014, Ghotloo, Haji

260 Mollahoseini et al. 2015); however, it has become increasingly apparent that both of these genes

261 are important virulence factors. AAP3 is upregulated in response to arginine shortages in host

262 macrophages, and SODB1 deficient L. chagasi parasites demonstrate impaired survival in host

263 macrophages (Plewes, Barr et al. 2003, Castilho-Martins, Laranjeira da Silva et al. 2011, Goldman-

264 Pinkovich, Balno et al. 2016, Muxel, Laranjeira-Silva et al. 2017). This raises concern about the

265 reliability of the assays during experimental manipulation; particularly in light of Leishmania spp.

266 regulating gene expression through copy number variation (Laffitte, Leprohon et al. 2016,

267 Iantorno, Durrant et al. 2017).

268 Amplifying the gene target DNA polymerase alpha has similar characteristics to the

269 DRBD3 assay. It is another example of single copy gene, which can be amplified at high efficiency

270 with a limit of detection of $100 \mathrm{fg}$ (Bretagne, Durand et al. 2001, Prina, Roux et al. 2007). 
271 Therefore, for monitoring parasite burden accurately and precisely in animal models, the DRBD3

272 qPCR assay and the DNA polymerase alpha assay (Prina, Roux et al. 2007) fulfill the optimal

273 characteristics.

274

275 Discussion

276 Animal models of leishmaniasis have proven to be valuable in understanding basic

277 immunological concepts, and will be critical in future drug development programs to control this

278 neglected tropical disease. The ability to accurately monitor parasite survival and replication in

279 these models is paramount to properly understanding the biology of infection and monitoring the

280 effectiveness of novel therapeutic interventions. The development of qPCR assays for parasite

281 quantification has been widespread for clinical applications; however, there is no standardized

282 qPCR protocol that is optimized specifically for use in animal models. Here we developed a novel

283 touchdown qPCR assay of the single copy housekeeping gene, DRBD3, which has a limit of

284 detection that rivals existing protocols while not being subject to changes in sequence or copy

285 number that multicopy genes or genes required for virulence would be subject to.

286 Accurate measurement of parasite burden by qPCR is important for distinguishing between

287 host and parasite mediated pathology. Disease progression of Leishmania animal models is

288 traditionally done by a combination of measuring footpad thickness or directly measuring parasites

289 through limiting dilution assays. Measuring the inflammation that results from infection by

290 swelling at the site of inoculation is a valuable way to monitor severity of disease, but it is unable

291 to distinguish between damage caused by overgrowth of the parasite and damage caused by

292 dysregulated host derived inflammation. Studies showing that parasite growth does not perfectly

293 correlate with lesion size, demonstrate the importance of distinguishing between parasite and host 
294 mediated pathology (Hill, North et al. 1983, Bretagne, Durand et al. 2001). This is particularly

295 relevant in the context of leishmaniasis where it is well documented that distinct disease

296 manifestations are caused by both extremes of this spectrum (Scott and Novais 2016). Limiting

297 dilution assays are useful for enumerating parasite burden, but are costly in terms of time and

298 resources. Of note, the qPCR assay described here does require access to a real-time PCR

299 thermocycler. However, given access to this equipment the protocol is significantly faster than the

300 approximately 1 week required for parasites to grow using the LDA. After the initial overnight

301 incubation used to isolate parasite DNA, using a qPCR approach to amplify parasite DNA allows

302 determination of parasite burden in as little as 2-4 hours.

The single copy gene DRBD3 is highly conserved across Leishmania spp., and the primers

304

305

306

307

308

309

310

311

312

313

314

315

used in this assay bind to a region of DNA over which $90 \%$ of the residues are completely conserved. However, it should be noted that the divergence of the 2 nucleotide positions occurs at the division between the Leishmania leishmania and Leishmania viannia subgenera. The primers reported here are $100 \%$ conserved within the leishmania subgenus. Within the viannia subgenus, the sequences are also $100 \%$ conserved, and are only altered at two sites between the two genera for each primer. We demonstrate that despite these two divergent sites, the described primers work with high efficiency for parasites in the viannia subgenus (L. panamensis, see Figure 1D). Further, the primers described here with optimal characteristics for parasite quantification in animal models could be adapted for use in developing iterations of PCR-based technology, such as PCRELISA(Kobets, Badalova et al. 2010), in order to further improve sensitivity and capacity of the assay. The DRBD3 qPCR assay for Leishmania quantification is therefore also likely to be a robust assay for labs in that validation of the assay one time will allow for use in a wide range of studies 
modeling parasites with distinct disease phenotypes and from diverse geographic and evolutionary

317

318

319

320

321

322

323

324

325

326

327

328

329

330

331

332

333

334

335

336

337

338

339

340

341

342

343

344

345

346

347

348

349

backgrounds.

\section{Conclusions}

Careful consideration of protocols used to quantify parasite burden in experimental models of leishmaniasis is essential to fully understand host-parasite interactions and for assessing the efficacy of novel therapeutic interventions. The DRBD3 assay described here will allow for consistent detection of parasites in an unbiased manner with a low limit of detection, facilitating discoveries in basic science and improvements in leishmaniasis treatment.

\section{$\underline{\text { References }}$}

Akopyants, N. S., R. S. Matlib, E. N. Bukanova, M. R. Smeds, B. H. Brownstein, G. D. Stormo and S. M. Beverley (2004). "Expression profiling using random genomic DNA microarrays identifies differentially expressed genes associated with three major developmental stages of the protozoan parasite Leishmania major." Mol Biochem Parasitol 136(1): 71-86.

Alvar, J., I. D. Velez, C. Bern, M. Herrero, P. Desjeux, J. Cano, J. Jannin, M. den Boer and W. H. O. L. C. Team (2012). "Leishmaniasis worldwide and global estimates of its incidence." PLoS One 7(5): e35671.

Aoki, J. I., S. M. Muxel, R. A. Zampieri, M. F. Laranjeira-Silva, K. E. Muller, A. H. Nerland and L. M. Floeter-Winter (2017). "RNA-seq transcriptional profiling of Leishmania amazonensis reveals an arginase-dependent gene expression regulation." PLoS NegI Trop Dis 11(10): e0006026.

Aslett, M., C. Aurrecoechea, M. Berriman, J. Brestelli, B. P. Brunk, M. Carrington, D. P. Depledge, S. Fischer, B. Gajria, X. Gao, M. J. Gardner, A. Gingle, G. Grant, O. S. Harb, M. Heiges, C. HertzFowler, R. Houston, F. Innamorato, J. lodice, J. C. Kissinger, E. Kraemer, W. Li, F. J. Logan, J. A. Miller, S. Mitra, P. J. Myler, V. Nayak, C. Pennington, I. Phan, D. F. Pinney, G. Ramasamy, M. B. Rogers, D. S. Roos, C. Ross, D. Sivam, D. F. Smith, G. Srinivasamoorthy, C. J. Stoeckert, Jr., S. Subramanian, R. Thibodeau, A. Tivey, C. Treatman, G. Velarde and H. Wang (2010). "TriTrypDB: a functional genomic resource for the Trypanosomatidae." Nucleic Acids Res 38(Database issue): D457-462.

Bailey, F., K. Mondragon-Shem, P. Hotez, J. A. Ruiz-Postigo, W. Al-Salem, A. Acosta-Serrano and D. H. Molyneux (2017). "A new perspective on cutaneous leishmaniasis-Implications for global prevalence and burden of disease estimates." PLoS Negl Trop Dis 11(8): e0005739.

Bensoussan, E., A. Nasereddin, F. Jonas, L. F. Schnur and C. L. Jaffe (2006). "Comparison of PCR

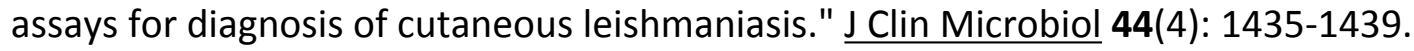


350 Bretagne, S., R. Durand, M. Olivi, J. F. Garin, A. Sulahian, D. Rivollet, M. Vidaud and M. Deniau 351 (2001). "Real-time PCR as a new tool for quantifying Leishmania infantum in liver in infected 352 mice." Clin Diagn Lab Immunol 8(4): 828-831.

353 Calvo-Alvarez, E., N. A. Guerrero, R. Alvarez-Velilla, C. F. Prada, J. M. Requena, C. Punzon, M. A. 354 Llamas, F. J. Arevalo, L. Rivas, M. Fresno, Y. Perez-Pertejo, R. Balana-Fouce and R. M. Reguera 355 (2012). "Appraisal of a Leishmania major strain stably expressing mCherry fluorescent protein for 356 both in vitro and in vivo studies of potential drugs and vaccine against cutaneous leishmaniasis." 357 PLoS Negl Trop Dis 6(11): e1927.

358 Castilho-Martins, E. A., M. F. Laranjeira da Silva, M. G. dos Santos, S. M. Muxel and L. M. Floeter359

360

361 Winter (2011). "Axenic Leishmania amazonensis promastigotes sense both the external and internal arginine pool distinctly regulating the two transporter-coding genes." PLoS One 6(11): e27818.

362

Croan, D. G., D. A. Morrison and J. T. Ellis (1997). "Evolution of the genus Leishmania revealed by 363 364 comparison of DNA and RNA polymerase gene sequences." Mol Biochem Parasitol 89(2): 149159.

365 Crooks, G. E., G. Hon, J. M. Chandonia and S. E. Brenner (2004). "WebLogo: A sequence logo 366 generator." Genome Research 14(6): 1188-1190.

368

369

370 Cruz, I., A. Millet, E. Carrillo, M. Chenik, P. Salotra, S. Verma, N. Veland, M. Jara, V. Adaui, C. Castrillon, J. Arevalo, J. Moreno and C. Canavate (2013). "An approach for interlaboratory comparison of conventional and real-time PCR assays for diagnosis of human leishmaniasis." Exp Parasitol 134(3): 281-289.

371 Cupolillo, E., G. Grimaldi Junior, H. Momen and S. M. Beverley (1995). "Intergenic region typing

372

373 (IRT): a rapid molecular approach to the characterization and evolution of Leishmania." Mol Biochem Parasitol 73(1-2): 145-155.

374 DALYs, G. B. D. and H. Collaborators (2017). "Global, regional, and national disability-adjusted life-years (DALYs) for 333 diseases and injuries and healthy life expectancy (HALE) for 195 countries and territories, 1990-2016: a systematic analysis for the Global Burden of Disease Study 2016." Lancet 390(10100): 1260-1344.

378 Das, A., V. Bellofatto, J. Rosenfeld, M. Carrington, R. Romero-Zaliz, C. del Val and A. M. Estevez 379 (2015). "High throughput sequencing analysis of Trypanosoma brucei DRBD3/PTB1-bound 380 mRNAs." Mol Biochem Parasitol 199(1-2): 1-4.

381 Fernandez-Moya, S. M., A. Garcia-Perez, S. Kramer, M. Carrington and A. M. Estevez (2012). 382 "Alterations in DRBD3 ribonucleoprotein complexes in response to stress in Trypanosoma 383 brucei." PLoS One 7(11): e48870.

384 Folgueira, C., C. Canavate, C. Chicharro and J. M. Requena (2007). "Genomic organization and 385 expression of the HSP70 locus in New and Old World Leishmania species." Parasitology 134(Pt 3): 386 369-377.

387 Ghotloo, S., M. Haji Mollahoseini, A. Najafi and F. Yeganeh (2015). "Comparison of Parasite 388 Burden Using Real-Time Polymerase Chain Reaction Assay and Limiting Dilution Assay in 389 Leishmania major Infected Mouse." Iran J Parasitol 10(4): 571-576.

390 Goldman-Pinkovich, A., C. Balno, R. Strasser, M. Zeituni-Molad, K. Bendelak, D. Rentsch, M. 391 Ephros, M. Wiese, A. Jardim, P. J. Myler and D. Zilberstein (2016). "An Arginine Deprivation 392 Response Pathway Is Induced in Leishmania during Macrophage Invasion." PLoS Pathog 12(4): 393 e1005494. 
394

395

396

397

398

399

400

401

402

403

404

405

406

407

408

409

410

411

412

413

414

415

416

417

418

419

420

421

422

423

424

425

426

427

428

429

430

431

432

433

434

435

436

437

Heinzel, F. P., M. D. Sadick, B. J. Holaday, R. L. Coffman and R. M. Locksley (1989). "Reciprocal expression of interferon gamma or interleukin 4 during the resolution or progression of murine leishmaniasis. Evidence for expansion of distinct helper T cell subsets." J Exp Med 169(1): 59-72. Hill, J. O., R. J. North and F. M. Collins (1983). "Advantages of measuring changes in the number of viable parasites in murine models of experimental cutaneous leishmaniasis." Infect Immun 39(3): 1087-1094.

lantorno, S. A., C. Durrant, A. Khan, M. J. Sanders, S. M. Beverley, W. C. Warren, M. Berriman, D. L. Sacks, J. A. Cotton and M. E. Grigg (2017). "Gene Expression in Leishmania Is Regulated Predominantly by Gene Dosage." MBio 8(5).

Ivens, A. C., C. S. Peacock, E. A. Worthey, L. Murphy, G. Aggarwal, M. Berriman, E. Sisk, M. A. Rajandream, E. Adlem, R. Aert, A. Anupama, Z. Apostolou, P. Attipoe, N. Bason, C. Bauser, A. Beck, S. M. Beverley, G. Bianchettin, K. Borzym, G. Bothe, C. V. Bruschi, M. Collins, E. Cadag, L. Ciarloni, C. Clayton, R. M. Coulson, A. Cronin, A. K. Cruz, R. M. Davies, J. De Gaudenzi, D. E. Dobson, A. Duesterhoeft, G. Fazelina, N. Fosker, A. C. Frasch, A. Fraser, M. Fuchs, C. Gabel, A. Goble, A. Goffeau, D. Harris, C. Hertz-Fowler, H. Hilbert, D. Horn, Y. Huang, S. Klages, A. Knights, M. Kube, N. Larke, L. Litvin, A. Lord, T. Louie, M. Marra, D. Masuy, K. Matthews, S. Michaeli, J. C. Mottram, S. Muller-Auer, H. Munden, S. Nelson, H. Norbertczak, K. Oliver, S. O'Neil, M. Pentony, T. M. Pohl, C. Price, B. Purnelle, M. A. Quail, E. Rabbinowitsch, R. Reinhardt, M. Rieger, J. Rinta, J. Robben, L. Robertson, J. C. Ruiz, S. Rutter, D. Saunders, M. Schafer, J. Schein, D. C. Schwartz, K. Seeger, A. Seyler, S. Sharp, H. Shin, D. Sivam, R. Squares, S. Squares, V. Tosato, C. Vogt, G. Volckaert, R. Wambutt, T. Warren, H. Wedler, J. Woodward, S. Zhou, W. Zimmermann, D. F. Smith, J. M. Blackwell, K. D. Stuart, B. Barrell and P. J. Myler (2005). "The genome of the kinetoplastid parasite, Leishmania major." Science 309(5733): 436-442.

Jara, M., V. Adaui, B. M. Valencia, D. Martinez, M. Alba, C. Castrillon, M. Cruz, I. Cruz, G. Van der Auwera, A. Llanos-Cuentas, J. C. Dujardin and J. Arevalo (2013). "Real-time PCR assay for detection and quantification of Leishmania (Viannia) organisms in skin and mucosal lesions: exploratory study of parasite load and clinical parameters." J Clin Microbiol 51(6): 1826-1833.

Kobets, T., J. Badalova, I. Grekov, H. Havelkova, M. Svobodova and M. Lipoldova (2010). "Leishmania parasite detection and quantification using PCR-ELISA." Nat Protoc 5(6): 1074-1080. Laffitte, M. N., P. Leprohon, B. Papadopoulou and M. Ouellette (2016). "Plasticity of the Leishmania genome leading to gene copy number variations and drug resistance." F1000Res 5: 2350.

Leifso, K., G. Cohen-Freue, N. Dogra, A. Murray and W. R. McMaster (2007). "Genomic and proteomic expression analysis of Leishmania promastigote and amastigote life stages: the Leishmania genome is constitutively expressed." Mol Biochem Parasitol 152(1): 35-46.

Leon, C. M., M. Munoz, C. Hernandez, M. S. Ayala, C. Florez, A. Teheran, J. R. Cubides and J. D. Ramirez (2017). "Analytical Performance of Four Polymerase Chain Reaction (PCR) and Real Time PCR (qPCR) Assays for the Detection of Six Leishmania Species DNA in Colombia." Front Microbiol 8: 1907.

Mary, C., F. Faraut, L. Lascombe and H. Dumon (2004). "Quantification of Leishmania infantum DNA by a real-time PCR assay with high sensitivity." J Clin Microbiol 42(11): 5249-5255.

McWilliam, H., W. Z. Li, M. Uludag, S. Squizzato, Y. M. Park, N. Buso, A. P. Cowley and R. Lopez (2013). "Analysis Tool Web Services from the EMBL-EBI." Nucleic Acids Research 41(W1): W597W600. 
438 Michel, G., B. Ferrua, T. Lang, M. P. Maddugoda, P. Munro, C. Pomares, E. Lemichez and P. Marty 439 (2011). "Luciferase-expressing Leishmania infantum allows the monitoring of amastigote 440 population size, in vivo, ex vivo and in vitro." PLoS Negl Trop Dis 5(9): e1323.

441 Muxel, S. M., M. F. Laranjeira-Silva, R. A. Zampieri and L. M. Floeter-Winter (2017). "Leishmania 442 (Leishmania) amazonensis induces macrophage miR-294 and miR-721 expression and modulates 443 infection by targeting NOS2 and L-arginine metabolism." Sci Rep 7: 44141.

444 Plewes, K. A., S. D. Barr and L. Gedamu (2003). "Iron superoxide dismutases targeted to the 445 glycosomes of Leishmania chagasi are important for survival." Infect Immun 71(10): 5910-5920. 446 Ponte-Sucre, A., F. Gamarro, J. C. Dujardin, M. P. Barrett, R. Lopez-Velez, R. Garcia-Hernandez, A. 447 W. Pountain, R. Mwenechanya and B. Papadopoulou (2017). "Drug resistance and treatment 448 failure in leishmaniasis: A 21st century challenge." PLoS Negl Trop Dis 11(12): e0006052.

449 Prina, E., E. Roux, D. Mattei and G. Milon (2007). "Leishmania DNA is rapidly degraded following 450 parasite death: an analysis by microscopy and real-time PCR." Microbes Infect 9(11): 1307-1315. 451 Rogers, M. B., J. D. Hilley, N. J. Dickens, J. Wilkes, P. A. Bates, D. P. Depledge, D. Harris, Y. Her, P. 452 Herzyk, H. Imamura, T. D. Otto, M. Sanders, K. Seeger, J. C. Dujardin, M. Berriman, D. F. Smith, C. 453 Hertz-Fowler and J. C. Mottram (2011). "Chromosome and gene copy number variation allow 454 major structural change between species and strains of Leishmania." Genome Res 21(12): 21294552142.

456 Roy, G., C. Dumas, D. Sereno, Y. Wu, A. K. Singh, M. J. Tremblay, M. Ouellette, M. Olivier and B. 457 Papadopoulou (2000). "Episomal and stable expression of the luciferase reporter gene for 458 quantifying Leishmania spp. infections in macrophages and in animal models." Mol Biochem 459 Parasitol 110(2): 195-206.

460 Sacks, D. L. and P. C. Melby (2001). "Animal models for the analysis of immune responses to 461 leishmaniasis." Curr Protoc Immunol Chapter 19: Unit 1912.

462 Scott, P., P. Natovitz, R. L. Coffman, E. Pearce and A. Sher (1988). "CD4+ T cell subsets in 463 experimental cutaneous leishmaniasis." Mem Inst Oswaldo Cruz 83 Suppl 1: 256-259.

464 Scott, P. and F. O. Novais (2016). "Cutaneous leishmaniasis: immune responses in protection and 465 pathogenesis." Nat Rev Immunol 16(9): 581-592.

466 Scott, P. and F. O. Novais (2016). "Cutaneous leishmaniasis: immune responses in protection and 467 pathogenesis." Nat Rev Immunol.

468 Tellevik, M. G., K. E. Muller, K. R. Lokken and A. H. Nerland (2014). "Detection of a broad range 469 of Leishmania species and determination of parasite load of infected mouse by real-time PCR 470 targeting the arginine permease gene AAP3." Acta Trop 137: 99-104.

471 Torres-Machorro, A. L., R. Hernandez, A. M. Cevallos and I. Lopez-Villasenor (2010). "Ribosomal 472 RNA genes in eukaryotic microorganisms: witnesses of phylogeny?" FEMS Microbiol Rev 34(1): 473 59-86.

474 Vita, R., L. Zarebski, J. A. Greenbaum, H. Emami, I. Hoof, N. Salimi, R. Damle, A. Sette and B. Peters 475 (2010). "The immune epitope database 2.0." Nucleic Acids Res 38(Database issue): D854-862.

476 Weirather, J. L., S. M. Jeronimo, S. Gautam, S. Sundar, M. Kang, M. A. Kurtz, R. Haque, A. Schriefer, 477 S. Talhari, E. M. Carvalho, J. E. Donelson and M. E. Wilson (2011). "Serial quantitative PCR assay 478 for detection, species discrimination, and quantification of Leishmania spp. in human samples." 479 J Clin Microbiol 49(11): 3892-3904. 
480 Wortmann, G., L. Hochberg, H. H. Houng, C. Sweeney, M. Zapor, N. Aronson, P. Weina and C. F. 481 Ockenhouse (2005). "Rapid identification of Leishmania complexes by a real-time PCR assay." Am 482 J Trop Med Hyg 73(6): 999-1004.

483 Ye, J., G. Coulouris, I. Zaretskaya, I. Cutcutache, S. Rozen and T. L. Madden (2012). "Primer-BLAST: 484 a tool to design target-specific primers for polymerase chain reaction." BMC Bioinformatics 13: 485134.

486 Yurchenko, V. Y., E. M. Merzlyak, A. A. Kolesnikov, L. P. Martinkina and Y. Y. Vengerov (1999). 487 "Structure of Leishmania minicircle kinetoplast DNA classes." J Clin Microbiol 37(5): 1656-1657. 488 


\section{Figure 1}

Primer design and optimization of DRBD3 based qPCR for parasite quantification.

A) Multisequence alignment based on 11 homologous sequences to $L$. major Sd. DRBD3 found using NCBI Blast B) Primer sequences and cycling parameters used. C). DRBD3 primers amplify a 140bp product specifically. Product visualized with ethidium bromide staining of a $1 \%$ agarose gel run and compared to New England BioLabs 100bp ladder. D) DRBD3 primers amplify diverse Leishmania spp. A representative plot of $\mathrm{C} t$ value vs log dilution of parasite burden is shown. The average primer efficiency ( \pm standard error of the mean) is indicated in parentheses for the following species: L. major Seidman $(n=6), L$. major Friedlin $(n=5), L$. venezuelensis $(n=5)$, L. panamensis $(n=3)$, and L. donovani $(n=3)$.
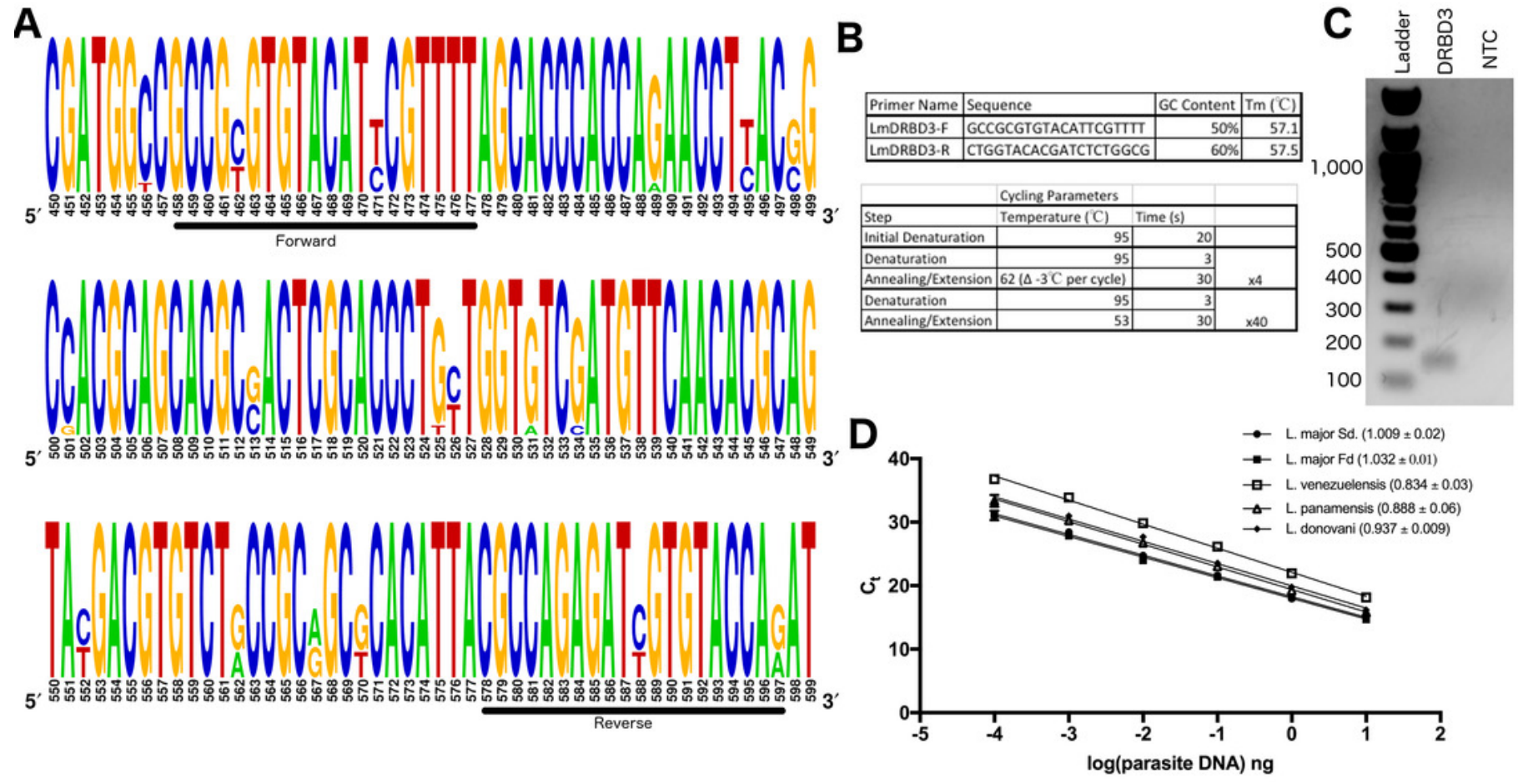


\section{Figure 2}

DRBD3 primers are able to assess parasite burden from infected mouse tissue.

A-B) Representative amplification plots and melt curve analysis from the draining lymph nodes of mice at 34 days post infection. Parasites amplified from infected tissue had melting temperatures corresponding with the expected $84.7^{\circ} \mathrm{C}$. DNA from uninfected tissue resulted in non-specific amplification at later cycles with melt temps outside of $84.7 \pm 0.5^{\circ} \mathrm{C}$. Samples that generated any product outside of this range were classified as having no leishmania specific amplification. C) Quantification of total parasites in the draining popliteal lymph nodes and infected footpads at 1 and 34 days post infection with L. major Seidman (Sd.). At 1 day post infection, 5 lymph node samples had no leishmania specific amplification and are not plotted. All samples from 34 days post infection had detectable leishmania specific amplification. D) DRBD3 quantification provides insights to disease pathogenesis in L. major Freidlin ( $F d$.) and $L$. venezuelensis infections. Comparison of footpad thickness and parasite burden in the draining lymph nodes and infected footpads at 36 days post infection. 4 lymph node samples and 1 footpad samples had no leishmania specific amplification and are therefore not plotted. P- values calculated by parametric Students T-test. 


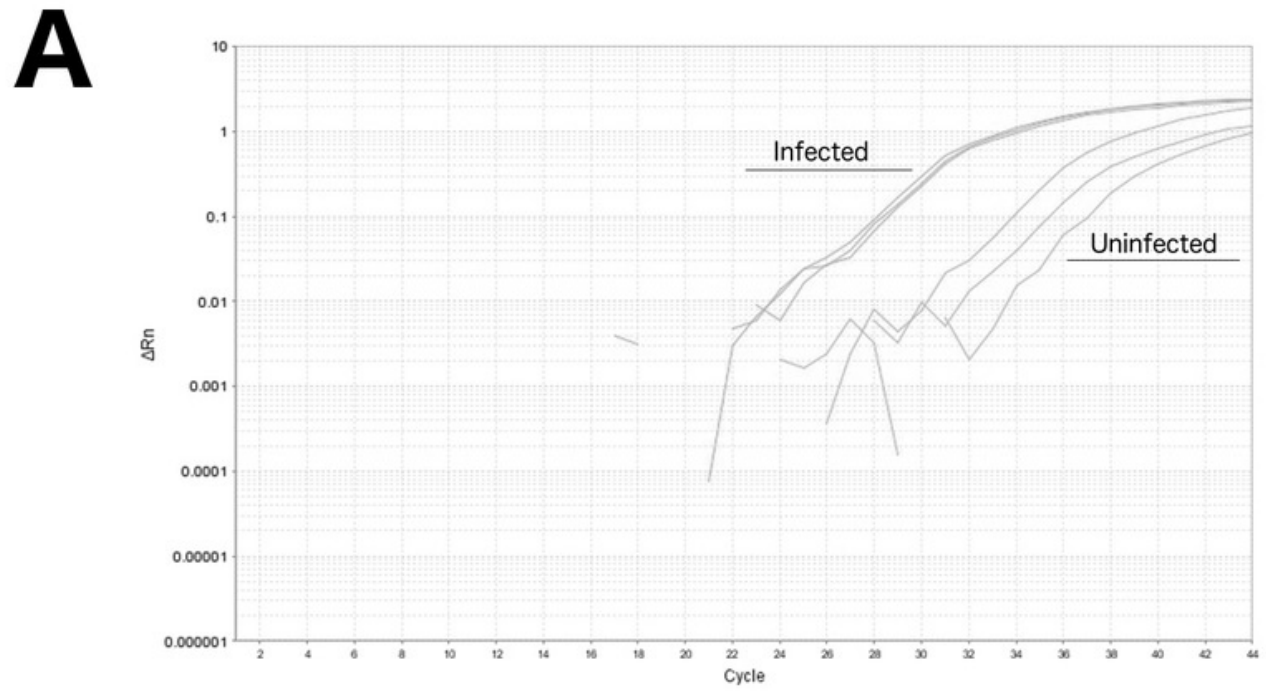

B
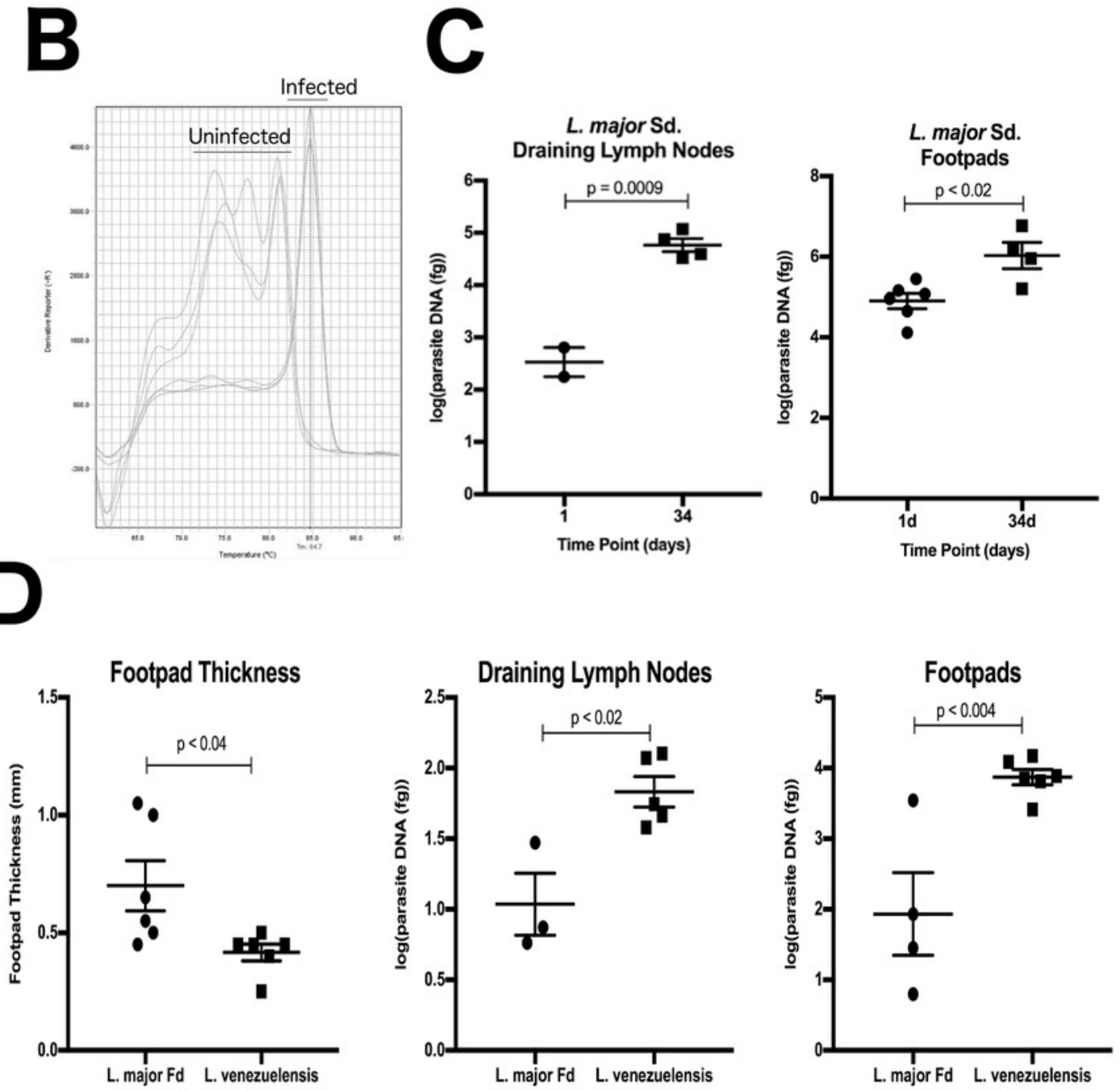


\section{Table $\mathbf{1}$ (on next page)}

Comparison of described targets for qPCR based quantification of parasite burden in humans and animals.

(Bretagne, Durand et al. 2001, Nicolas, Prina et al. 2002, Bossolasco, Gaiera et al. 2003, Schonian, Nasereddin et al. 2003, Schulz, Mellenthin et al. 2003, Mary, Faraut et al. 2004, Wortmann, Hochberg et al. 2005, Prina, Roux et al. 2007, Castilho, Camargo et al. 2008, Deborggraeve, Laurent et al. 2008, van der Meide, Guerra et al. 2008, de Paiva Cavalcanti, Felinto de Brito et al. 2009, Bezerra-Vasconcelos, Melo et al. 2011, Jara, Adaui et al. 2013, Srivastava, Sweat et al. 2013, Toz, Culha et al. 2013, Tellevik, Muller et al. 2014, Ghotloo, Haji Mollahoseini et al. 2015, Zampieri, Laranjeira-Silva et al. 2016) 


\begin{tabular}{|c|c|c|c|c|c|c|}
\hline Gene Target & Copy Number & Sybr/TaqMan? & Primer Efficiency & Limit of Quantification & Amplified from Animal Sample & Citation(s) \\
\hline Arginine Permease (AAP3) & 1 & TaqMan & 1.051 & $10 \mathrm{fg}$ & Mice & Tellevik (2013) \\
\hline 18s rRNA & 63-166 & TaqMan/Sybr & $0.831-0.942$ & $10 \mathrm{fg}$ & Humans; Sandflies & $\begin{array}{l}\text { Shulz (2003); van der Meide (2008); Bossolasco (2003); } \\
\text { Prina (2007); Deborggraeve (2013); Bezerra-Vasconcelos (2011) }\end{array}$ \\
\hline DNA Polymerase & 1 & TaqMan/Sybr & $0.934-0.9941$ & $\begin{array}{c}100 \mathrm{fg} \\
0-500 \text { parasites per } 500 \text { hepatic cells }\end{array}$ & Mice & Bretagne (2001); Prina (2007) \\
\hline Glucose 6 Phosphate Dehydrogenase (G6PD) & 1 & TaqMan/Sybr & $0.504-0.957$ & $\begin{array}{c}100 \mathrm{fg} \\
20 \text { gene copies per rxn }\end{array}$ & Humans; Mice & Castilho (2008); Prina (2007) \\
\hline Glucosephopsphate isomerase (GPI) & 1 & TaqMan & NR & $5.6 \mathrm{pg}$ & Humans & Wortmann (2005) \\
\hline Heat Shock Protein 70 (HSP70) & $5-7$ & SYTO9 & $0.9237-0.9723$ & $50 \mathrm{fg}$ & Humans; Mice; Sandflies & Zampieri (2016) \\
\hline Internal Transcribed Spacer (ITS1) & $20-200$ & TaqMan & NR & 0.2 parasites per sample & Humans; Dogs & Toz (2013); Shonian (2003) \\
\hline Kinetoplast Minicircle DNA (kDNA) & $500-1000$ & TaqMan/Sybr & $0.79-1.05$ & $10 \mathrm{fg}$ & $\begin{array}{l}\text { Humans; Dogs; Hampsters; } \\
\text { Mice; Sandflies }\end{array}$ & $\begin{array}{c}\text { Nicolas (2002); Cavalcanti (2008); Francino (2006); } \\
\text { Mary (2004); Jara (2013); Srivastava (2013); } \\
\text { Bezerra-Vasconcelos (2011) }\end{array}$ \\
\hline RNA Binding Protein (DRBD3) & 1 & Sybr & 0.971 & $100 \mathrm{fg}$ & Mice & This report \\
\hline Superoxide Dismutase B1 (SODB1) & 1 & Sybr & 0.91 & 50 parasites & Mice & Ghotloo (2015) \\
\hline
\end{tabular}

